\title{
Weak homoclinic solutions to discrete nonlinear problems of Kirchhoff type with variable exponents
}

\author{
Aboudramane Guiro, Idrissa Ibrango and Stanislas Ouaro \\ LAboratoire de Mathématiques et Informatique (LAMI) \\ 1 UFR, Sciences et Techniques, Universit Nazi BONI \\ 01 BP 1091 Bobo-Dioulasso, 01 Bobo Dioulasso, Burkina Faso \\ 2 UFR. Sciences Exactes et Appliques, Universit Ouaga \\ I Pr Joseph KI-ZERBO, 03 BP 7021 Ouaga 03, Ouagadougou, Burkina Faso \\ abouguiro@yahoo.fr, ibrango2006@yahoo.fr, ibrango2006@yahoo.fr, ouaro@yahoo.fr
}

\begin{abstract}
In this paper, we prove the existence of weak homoclinic solutions for discrete nonlinear problems of Kirchhoff type. The proof of the main result is based on a minimization method. As extension, we prove the existence result of weak homoclinic solutions for more general data depending on the solutions.
\end{abstract}

\section{RESUMEN}

En este artículo, probamos la existencia de soluciones homoclínicas débiles para problemas discretos no-lineales de tipo Kirchhoff. La demostración del resultado principal está basado en un método de minimización. Como extensión, probamos la existencia de soluciones homoclínicas débiles para datos más generales dependiendo de las soluciones.

2010 AMS Mathematics Subject Classification: 47A75, 35B38, 35P30, 34L05, 34L30. 


\section{Introduction}

In this paper, we study the following nonlinear discrete anisotropic problem

$$
(P)\left\{\begin{array}{l}
-M(A(k-1, \Delta u(k-1))) \Delta(a(k-1, \Delta u(k-1)))+|u(k)|^{p(k)-2} u(k)=f(k), k \in \mathbb{Z} \\
\lim _{|k| \rightarrow \infty} u(k)=0,
\end{array}\right.
$$

where $\Delta \mathfrak{u}(k)=\mathfrak{u}(k+1)-\mathfrak{u}(k)$ is the forward difference operator.

Note that difference equations can be seen as a discrete counterpart of partial difference equations and are usually studied in connection with numerical analysis. In this way, the operator in problem (1.1)

$$
\Delta(\mathrm{a}(\mathrm{k}-1, \Delta \mathrm{u}(\mathrm{k}-1)))
$$

can be seen as a discrete counterpart of the anisotropic operator

$$
\sum_{i=1}^{N} \frac{\partial}{\partial x_{i}} a\left(x, \frac{\partial}{\partial x_{i}} u\right)
$$

The first study in that direction for constant exponents was done by Cabada et al. [2] and for variable exponent by Mihailescu et al. [10] (see also [3]). In [3], the authors studied the following problem

$$
\left\{\begin{array}{l}
-\Delta(a(k-1, \Delta u(k-1)))+|u(k)|^{p(k)-2} u(k)=f(k), \quad k \in \mathbb{Z} \\
\lim _{|k| \rightarrow+\infty} u(k)=0 .
\end{array}\right.
$$

They proved an existence result of weak homoclinic solution of (1.2).

In this paper we consider the same boundary conditions as in $[\underline{3}$, but the function

$$
M(A(k-1, \Delta u(k-1)))
$$

which appear in the left-hand side of problem (1.1) is more general than the one which appear in [3]. Indeed, if we take $M(t)=1$ in the problem (1.1), we obtain the probem studied by Guiro et als in $[3$.

To prove an existence result of problem (1.1), we define other new spaces and new associated norms and we adapt the classical minimization methods used for the study of anisotropic $\mathrm{PDE}_{s}$. The idea is to transfer the problem of the existence of solutions for (1.1) into the problem of the existence of a minimizer for some associated energy functional.

The remaining part of this paper is organized as follows: section 2 is devoted to mathematical preliminaries. The main existence result is stated and proved in section 3. Finally, in section 4, we discuss some extensions. 


\section{Preliminaries}

We use the notations

$$
p^{+}=\sup _{k \in \mathbb{Z}} p(k) \text { and } p^{-}=\inf _{k \in \mathbb{Z}} p(k) .
$$

For the data $f$ and $a$, we assume the following:

$$
\begin{gathered}
\left\{\begin{array}{l}
a(., .): \mathbb{Z} \times \mathbb{R} \longrightarrow \mathbb{R} \text { and } A(., .): \mathbb{Z} \times \mathbb{R} \longrightarrow \mathbb{R} \text { with } \\
a(k, \xi)=\frac{\partial}{\partial \xi} A(k, \xi) \text { and } A(k, 0)=0, \quad \forall k \in \mathbb{Z} .
\end{array}\right. \\
|\xi|^{p(k)} \leq a(k, \xi) \xi \leq p(k) A(k, \xi), \quad \forall k \in \mathbb{Z} \text { and } \xi \in \mathbb{R} .
\end{gathered}
$$

There exists a positive constant $C_{1}$ such that

$$
|a(k, \xi)| \leq C_{1}\left(j(k)+|\xi|^{p(k)-1}\right),
$$

for all $k \in \mathbb{Z}$ and $\xi \in \mathbb{R}$, where $j \in \mathcal{L}^{p^{\prime}(.)}$ with $\frac{1}{p(k)}+\frac{1}{p^{\prime}(k)}=1$.

Let us take

$$
f \in \mathcal{L}^{p^{\prime}(\cdot)} .
$$

For $\xi, \eta \in \mathbb{R}$ with $\xi \neq \eta$, and for almost every $k \in \mathbb{Z}$,

$$
\left(a_{i}(x, \xi)-a_{i}(x, \eta)\right)(\xi-\eta) \geq 0 .
$$

Moreover, in this paper, we assume that the function

$$
\mathrm{p}: \mathbb{Z} \rightarrow(1,+\infty) \text { such that } 1<\mathrm{p}^{-}<\mathrm{p}(.)<\mathrm{p}^{+}<+\infty .
$$

We also assume that the function $M:(0,+\infty) \rightarrow(0,+\infty)$ is continuous, non decreasing and there exist two positive reals number $B_{1}, B_{2}$ such that $B_{1} \leq B_{2}$ and $\alpha \geq 1$ with

$$
B_{1} t^{\alpha-1} \leq M(t) \leq B_{2} t^{\alpha-1}, \text { for } t>0
$$

Let us define the functional spaces,

$$
\mathcal{L}^{p(.)}=\left\{u: \mathbb{Z} \longrightarrow \mathbb{R} \text { such that } \rho_{p(.)}(u):=\sum_{k \in \mathbb{Z}}|u(k)|^{p(k)}<\infty\right\}
$$

and

$$
\mathcal{W}_{\alpha}^{1, p(.)}=\left\{u: \mathbb{Z} \longrightarrow \mathbb{R} \text { such that } \rho_{1, \alpha, p(.)}(u):=\sum_{k \in \mathbb{Z}}|u(k)|^{p^{(k)}}+\left(\sum_{k \in \mathbb{Z}}|\Delta u(k)|^{p(k)}\right)^{\alpha}<\infty\right\} .
$$

We introduce in $\mathcal{L}^{p(.)}$ the Luxemburg norm

$$
\|\mathfrak{u}\|_{p(.)}:=\inf \left\{\lambda>0 \text { such that } \sum_{k \in \mathbb{Z}}\left|\frac{u(k)}{\lambda}\right|^{p(k)} \leq 1\right\},
$$

and we define, on the space $\mathcal{W}_{\alpha}^{1, p(.)}$, the norm

$$
\|u\|_{1, \alpha, p(.)}:=\inf \left\{\lambda>0 \text { such that } \sum_{k \in \mathbb{Z}}\left|\frac{u(k)}{\lambda}\right|^{p(k)}+\left(\sum_{k \in \mathbb{Z}}\left|\frac{\Delta u(k)}{\lambda}\right|^{p(k)}\right)^{\alpha} \leq 1\right\} .
$$




\section{Example 2.1.}

As example of functions which satsfies above assumptions, we have the following.

- $A(k, \xi)=\frac{1}{p(k)}|\xi|^{p(k)}$, where $a(k, \xi)=|\xi|^{p(k)-2} \xi, \forall k \in \mathbb{Z}$ and $\xi \in \mathbb{R}$,

- $A(k, \xi)=\frac{1}{p(k)}\left(\left(1+|\xi|^{2}\right)^{p(k) / 2}-1\right)$, where $a(k, \xi)=\left(1+|\xi|^{2}\right)^{(p(k)-2) / 2} \xi, \quad \forall k \in \mathbb{Z}, \xi \in \mathbb{R}$

and

- $\mathrm{B}(\mathrm{t})=\mathrm{ct}^{\alpha-1}$, for $\mathrm{t}>0$, where $\alpha \geq 1$ and $\mathrm{c}>0$.

Remark 2.2. If $u \in \mathcal{L}^{p^{(.)}}$, then $\lim _{|k| \rightarrow+\infty} u(k)=0$.

Indeed, if $u \in \mathcal{L}^{p(.)}$, then $\sum_{k \in \mathbb{Z}}|u(k)|^{p(k)}<+\infty$.

Let

$$
S_{1}=\{k \in \mathbb{Z} ;|u(k)|<1\} \text { and } S_{2}=\{k \in \mathbb{Z} ;|u(k)| \geq 1\}
$$

$\mathrm{S}_{2}$ is necessary a finite set and $|\mathfrak{u}(\mathrm{k})|<+\infty$ for any $\mathrm{k} \in \mathrm{S}_{2}$ since $\mathrm{u} \in \mathcal{L}^{\mathrm{p}(\text {.) }}$. As $S_{2}$ is a finite set, then $\sum_{k \in S_{2}}|u(k)|^{p^{+}}<+\infty$.

Therefore

$$
\sum_{k \in \mathbb{Z}}|\mathfrak{u}(k)|^{p^{+}}=\sum_{k \in S_{1}}|u(k)|^{p^{+}}+\sum_{k \in S_{2}}|\mathfrak{u}(k)|^{p^{+}}<+\infty
$$

Thus,

$$
\lim _{|k| \rightarrow+\infty} u(k)=0
$$

In the sequel, we will use the following result:

Proposition 2.3. ([3], Proposition 2.3). If $\mathrm{u} \in \mathcal{L}^{\mathrm{p}(.)}$ and $\mathrm{p}^{+}<\infty$, then the following properties hold:

(1) $\|u\|_{p(.)}<1 \Longrightarrow\|u\|_{p^{(.)}}^{p+} \leq \rho_{\mathfrak{p}(.)}(u) \leq\|u\|_{p^{(.)}}^{p^{-}} ;$

$(2)\|u\|_{p(.)}>1 \Longrightarrow\|u\|_{\mathfrak{p}(.)}^{p^{-}} \leq \rho_{\mathfrak{p}(.)}(u) \leq\|u\|_{\mathfrak{p}^{(.)}}^{\mathfrak{p}^{+}} ;$

(3) $\|\mathfrak{u}\|_{p(.)}<1(=1 ;>1) \Longleftrightarrow \rho_{\mathfrak{p}(.)}(\mathfrak{u})<1(=1 ;>1)$.

Theorem 2.4. ([3], Theorem 2.1). Let $u \in \mathcal{L}^{\mathrm{p}(.)}$ and $v \in \mathcal{L}^{\mathrm{q}(.)}$ such that $\frac{1}{\mathrm{p}(\mathrm{k})}+\frac{1}{\mathrm{q}(\mathrm{k})}=1$ for any $\mathrm{k}$ in $\mathbb{Z}$. Then

$$
\sum_{k \in \mathbb{Z}}|u v| \leq\left(\frac{1}{p^{-}}+\frac{1}{q^{-}}\right)\|u\|_{p(.)}\|v\|_{q(.)} .
$$


Proposition 2.5. ([10], Proposition 2.1). Let $\mathrm{q}: \mathbb{Z} \longrightarrow \mathbb{R}$ such that

$$
1<\mathrm{p}^{-} \leq \mathrm{p}^{+}<\mathrm{q}^{-} \leq \mathrm{q}^{+}
$$

Then

$$
\mathcal{L}^{p^{(\cdot)}} \subset \mathcal{L}^{\mathrm{q}(\cdot)}
$$

As in [3], we have the following result.

Proposition 2.6.

(1) $\rho_{1, \alpha, \mathfrak{p}(.)}(u+v) \leq 2^{\alpha p^{+}-1}\left(\rho_{1, \alpha, \mathfrak{p}(.)}(u)+\rho_{1, \alpha, p(.)}(v)\right), \quad \forall u, v \in \mathcal{W}_{\alpha}^{1, \mathfrak{p}(.)}$

(2) Let $u$ in $\mathcal{W}_{\alpha}^{1, p(.)}$.

i) If $\underline{\lambda>1}$, we have

$$
\lambda^{p^{-}} \rho_{1, \alpha, p(.)}(u) \leq \rho_{1, \alpha, p(.)}(\lambda u) \leq \lambda^{\alpha p^{+}} \rho_{1, \alpha, p(.)}(u) .
$$

ii) If $\underline{0<\lambda<1}$, we have

$$
\lambda^{\alpha p^{+}} \rho_{1, \alpha, p(.)}(u) \leq \rho_{1, \alpha, p(.)}(\lambda u) \leq \lambda^{p^{-}} \rho_{1, \alpha, p(.)}(u) .
$$

Theorem 2.7. Let $u \in \mathcal{W}_{\alpha}^{1, \mathfrak{p}(.)} \backslash\{0\}$. Then $\|u\|_{1, \alpha, \mathfrak{p}(.)}=a$ if and only if $\rho_{1, \alpha, \mathfrak{p}(.)}(\mathfrak{u} / \mathfrak{a})=1$.

Proposition 2.8. If $\mathrm{u} \in \mathcal{W}_{\alpha}^{1, p(.)}$ and $\mathrm{p}^{+}<\infty$, then the following properties hold:

(1) $\|u\|_{1, \alpha, p(.)}<1 \Longrightarrow\|\mathfrak{u}\|_{1, \alpha, p(.)}^{\alpha \mathfrak{p}+} \leq \rho_{1, \alpha, \mathfrak{p}(.)}(\mathfrak{u}) \leq\|u\|_{1, \alpha, \mathfrak{p}(.)}^{\mathfrak{p}^{-}} ;$

$(2)\|u\|_{1, \alpha, p(.)}>1 \Longrightarrow\|u\|_{1, \alpha, p(.)}^{p-} \leq \rho_{1, \alpha, p(.)}(u) \leq\|u\|_{1, \alpha, \mathfrak{p}(.)}^{\alpha \mathfrak{p}^{+}} ;$

(3) $\|u\|_{1, \alpha, p(.)}<1(=1 ;>1) \Longleftrightarrow \rho_{1, \alpha, p(.)}(u)<1(=1 ;>1)$.

\section{Existence of weak homoclinic solutions}

In this section we investigate the existence of weak homoclinic solutions of problem (1.1).

The energy functional corresponding to problem (1.1) is defined as $\mathrm{J}: \mathcal{W}_{\alpha}^{1, p(.)} \longrightarrow \mathbb{R}$ such that

$$
J(u)=\widehat{M}\left(\sum_{k \in \mathbb{Z}} A(k-1, \Delta u(k-1))\right)+\sum_{k \in \mathbb{Z}} \frac{1}{p(k)}|u(k)|^{p(k)}-\sum_{k \in \mathbb{Z}} f(k) u(k),
$$

where $\widehat{M}(t)=\int_{0}^{t} M(s) d s$. 
Definition 3.1. A weak homoclinic solution of problem (1.1) is a function $u \in \mathcal{W}_{\alpha}^{1, p(.)}$ such that

$$
\left\{\begin{array}{l}
M\left(\sum_{k \in \mathbb{Z}} A(k-1, \Delta u(k-1))\right) \sum_{k \in \mathbb{Z}} a(k-1, \Delta u(k-1)) \Delta v(k-1) \\
+\sum_{k \in \mathbb{Z}}|u(k)|^{p(k)-2} u(k) v(k)=\sum_{k \in \mathbb{Z}} f(k) v(k)
\end{array}\right.
$$

for any $v \in \mathcal{W}_{\alpha}^{1, p(.)}$.

Hence the critical points of functionnal J are the weak solutions for problem (1.1).

The main result is the following:

Theorem 3.2. Assume that hypotheses (2.1)-2.7) hold. Then, there exists at least one weak homoclinic solution of problem (1.1).

Proof. We first present some basic properties of the functional J.

Proposition 3.3. The functional $\mathrm{J}$ is well defined on $\mathcal{W}_{\alpha}^{1, \mathrm{p}(.)}$ and is of class $\mathrm{C}^{1}\left(\mathcal{W}_{\alpha}^{1, \mathrm{p}(.)}, \mathbb{R}\right)$ with the derivative given by

$$
\left\{\begin{aligned}
\left\langle J^{\prime}(u), v\right\rangle= & M\left(\sum_{k \in \mathbb{Z}} A(k-1, \Delta u(k-1))\right) \sum_{k \in \mathbb{Z}} a(k-1, \Delta u(k-1)) \Delta v(k-1) \\
& +\sum_{k \in \mathbb{Z}}|u(k)|^{p(k)-2} u(k) v(k)-\sum_{k \in \mathbb{Z}} f(k) v(k),
\end{aligned}\right.
$$

for all $u, v \in \mathcal{W}_{\alpha}^{1, p(.)}$.

Indeed, we denote by

$$
I(u)=\widehat{M}\left(\sum_{k \in \mathbb{Z}} A(k-1, \Delta u(k-1))\right), \quad L(u)=\sum_{k \in \mathbb{Z}} \frac{1}{p(k)}|u(k)|^{p(k)} \text { and } \wedge(u)=\sum_{k \in \mathbb{Z}} f(k) u(k)
$$

We have, by using (2.7), that

$$
\begin{aligned}
& |\mathrm{I}(\mathrm{u})|=\left|\int_{0}^{\sum_{k \in \mathbb{Z}} A(k-1, \Delta u(k-1))} \mathrm{M}(\mathrm{t}) \mathrm{dt}\right| \\
& \leq B_{2}\left|\int_{0}^{\sum_{k \in \mathbb{Z}} A(k-1, \Delta u(k-1))} t^{\alpha-1} d t\right| \\
& \leq \frac{\mathrm{B}_{2}}{\alpha}\left(\sum_{\mathrm{k} \in \mathbb{Z}}|\mathrm{A}(\mathrm{k}-1, \Delta \mathrm{u}(\mathrm{k}-1))|\right)^{\alpha} \text {. }
\end{aligned}
$$


According to (2.1), (2.3) and the discrete Hlder type inequality, we write

$$
\begin{aligned}
\sum_{k \in \mathbb{Z}}|A(k-1, \Delta u(k-1))| & \leq \sum_{k \in \mathbb{Z}} \int_{0}^{\Delta u(k-1)}|a(k-1, t)| d t \\
& \leq C_{1} \sum_{k \in \mathbb{Z}}\left(j(k-1)+\frac{1}{p(k-1)}|\Delta u(k-1)|^{p(k-1)-1}\right) \Delta u(k-1) \\
& \leq C_{1} \sum_{k \in \mathbb{Z}} j(k-1)|\Delta u(k-1)|+\frac{C_{1}}{p^{-}} \sum_{k \in \mathbb{Z}}|\Delta u(k-1)|^{p(k-1)} \\
& \leq C_{1}\left(\frac{1}{q^{-}}+\frac{1}{p^{-}}\right)\|j\|_{q(.)}\|\Delta u\|_{p(.)}+\frac{C_{1}}{p^{-}}\|\Delta u\|_{p(.)} \\
& <+\infty,
\end{aligned}
$$

and we deduce that $|\mathrm{I}(\mathrm{u})|<+\infty$.

We have

$$
|\mathrm{L}(\mathrm{u})|=\left.\left|\sum_{\mathrm{k} \in \mathbb{Z}} \frac{1}{p(k)}\right| \mathfrak{u}(\mathrm{k})\right|^{p(k)}\left|\leq \frac{1}{p^{-}}\right| \sum_{k \in \mathbb{Z}}|\mathfrak{u}(k)|^{p(k)} \mid<+\infty
$$

and

$$
|\Lambda(u)|=\left|\sum_{k \in \mathbb{Z}} f(k) u(k)\right| \leq \sum_{k \in \mathbb{Z}}|f(k)||u(k)|<+\infty .
$$

Clearly, the functionals $I, L$ and $\Lambda$ are in $C^{1}\left(\mathcal{W}_{\alpha}^{1, p(.)}, \mathbb{R}\right)$.

Therefore, the functional $\mathrm{J}$ is well defined on $\mathcal{W}_{\alpha}^{1, p(.)}$ and is of $\operatorname{class} \mathrm{C}^{1}\left(\mathcal{W}_{\alpha}^{1, p(\cdot)}, \mathbb{R}\right)$.

In what follows we prove (3.3). Let $u, v \in \mathcal{W}_{\alpha}^{1, \mathfrak{p}(.)}$. We have

$$
\left\{\begin{array}{l}
\lim _{h \rightarrow 0^{+}} \frac{I(u+h v)-I(u)}{h} \\
=\lim _{h \rightarrow 0^{+}} \frac{\widehat{M}\left(\sum_{k \in \mathbb{Z}} A(k-1, \Delta u(k-1)+h \Delta v(k-1))\right)-\widehat{M}\left(\sum_{k \in \mathbb{Z}} A(k-1, \Delta u(k-1))\right)}{h} \\
=M\left(\sum_{k \in \mathbb{Z}} A(k-1, \Delta \mathfrak{u}(k-1))\right) \sum_{k \in \mathbb{Z}} a(k-1, \Delta u(k-1)) \Delta v(k-1) .
\end{array}\right.
$$

Let us denote $g_{h}=\frac{|u(k)+h v(k)|^{p(k)}-|u(k)|^{p(k)}}{p(k) h}$.

We have

$$
\sum_{k \in \mathbb{Z}}\left|g_{h}\right| \leq \frac{1}{p^{-} h} \sum_{k \in \mathbb{Z}}|u(k)|^{p(k)}+\frac{1}{p^{-} h} \sum_{k \in \mathbb{Z}}|u(k)+h v(k)|^{p(k)}<+\infty .
$$


Thus

$$
\begin{aligned}
& \lim _{h \rightarrow 0^{+}} \frac{L(u+h v)-L(u)}{h}=\lim _{h \rightarrow 0^{+}} \sum_{k \in \mathbb{Z}} \frac{|u(k)+h v(k)|^{p(k)}-|u(k)|^{p(k)}}{p(k) h} \\
& =\sum_{k \in \mathbb{Z}} \lim _{h \rightarrow 0^{+}} \frac{|u(k)+h v(k)|^{p(k)}-|u(k)|^{p(k)}}{p(k) h} \\
& =\sum_{k \in \mathbb{Z}}|u(k)|^{p(k)-2} u(k) v(k) \text {. }
\end{aligned}
$$

And

$$
\begin{aligned}
\lim _{h \rightarrow 0^{+}} \frac{\Lambda(u+h v)-\Lambda(u)}{h} & =\lim _{h \rightarrow 0^{+}} \sum_{k \in \mathbb{Z}} \frac{f(k)(u(k)+h v(k))-f(k) u(k)}{h} \\
& =\sum_{k \in \mathbb{Z}} f(k) v(k) .
\end{aligned}
$$

Proposition 3.4. The functional J is weakly lower semi-continuous.

Indeed, by (2.1), (2.5) and (2.7) we have that $J$ is convex. Thus, it is enough to show that $J$ is lower semi-continuous. For this, we fix $u \in \mathcal{W}_{\alpha}^{1, p(.)}$ and $\epsilon>0$. Since $\mathrm{J}$ is convex, we deduce that for any $v \in \mathcal{W}_{\alpha}^{1, p(.)}$,

$$
\begin{aligned}
J(v) & \geq J(u)+\left\langle J^{\prime}(u), v-u\right\rangle \\
& \geq J(u)+R(u, v)+S(u, v)+T(u, v),
\end{aligned}
$$

with

$$
R(u, v)=M\left(\sum_{k \in \mathbb{Z}} A(k-1, \Delta u(k-1))\right) \sum_{k \in \mathbb{Z}} a(k-1, \Delta u(k-1))(\Delta v(k-1)-\Delta u(k-1))
$$

and

$$
S(u, v)=\sum_{k \in \mathbb{Z}}|u(k)|^{p(k)-2} u(k)(v(k)-u(k)) \quad \text { and } \quad T(u, v)=\sum_{k \in \mathbb{Z}} f(k)(u(k)-v(k)) .
$$

Using the Hlder type inequality there exist tree non negative constants $C_{3}, C_{4}$ and $C_{5}$ such that

$$
\begin{aligned}
R(u, v) & \geq-M\left(\sum_{k \in \mathbb{Z}} A(k-1, \Delta u(k-1))\right) \sum_{k \in \mathbb{Z}}|a(k-1, \Delta u(k-1)) \| \Delta v(k-1)-\Delta u(k-1)| \\
& \geq-C_{3}\|\Delta u-\Delta v\|_{p}(.) \\
\geq & -C_{3}\|u-v\|_{1, \alpha, p}(.)
\end{aligned}
$$

and

$$
\mathrm{T}(\mathrm{u}, v) \geq-\mathrm{C}_{4}\|\mathrm{u}-v\|_{1, \alpha, p(.)}
$$


Also

$$
\begin{aligned}
S(u, v) & \geq-\sum_{k \in \mathbb{Z}}|u(k)|^{p(k)-1}|v(k)-u(k)| \\
& \geq-\left(\frac{1}{p^{-}}+\frac{1}{\left(p^{\prime}\right)^{-}}\right)\left|\left\|\left.u\right|^{p(.)-1}\right\|\right|_{p^{\prime}(.)}\|u-v\|_{p(.)} \\
& \geq-C_{5}\|u-v\|_{1, \alpha, p(.)} .
\end{aligned}
$$

Then, combining (3.4), (3.5) and (3.6), we get

$$
J(v) \geq J(u)-C_{6}\|u-v\|_{1, \alpha, p(.)}
$$

with $\mathrm{C}_{6}=\mathrm{C}_{3}+\mathrm{C}_{4}+\mathrm{C}_{5}$.

Finally for all $v \in \mathcal{W}_{\alpha}^{1, p(.)}$ with $\|v-u\|_{1, \alpha, p(.)}<\delta=\frac{\epsilon}{\mathrm{C}_{6}}$, we get

$$
J(v) \geq J(u)-\epsilon .
$$

Then $\mathrm{J}$ is lower semi-continuous and by corollary III.8 in [1, we conclude that $\mathrm{J}$ is weakly lower semi-continuous.

Proposition 3.5. The functional $\mathrm{J}$ is coercive and bounded from below.

Indeed, we have

$$
\begin{aligned}
J(u) & =\widehat{M}\left(\sum_{k \in \mathbb{Z}} A(k-1, \Delta u(k-1))\right)+\sum_{k \in \mathbb{Z}} \frac{1}{p(k)}|u(k)|^{p(k)}-\sum_{k \in \mathbb{Z}} f(k) u(k) \\
& \geq \frac{B_{1}}{\alpha}\left(\sum_{k \in \mathbb{Z}} A(k-1, \Delta u(k-1))\right)^{\alpha}+\frac{1}{p^{+}} \sum_{k \in \mathbb{Z}}|u(k)|^{p(k)}-C_{7}\|f\|_{p^{\prime}(.)}\|u\|_{p(.)} \\
& \geq \frac{B_{1}}{\alpha}\left(\sum_{k \in \mathbb{Z}} \frac{1}{p(k-1)}|\Delta u(k-1)|^{p(k-1)}\right)^{\alpha}+\frac{1}{p^{+}} \sum_{k \in \mathbb{Z}}|u(k)|^{p(k)}-C_{7}^{\prime}\|u\|_{1, \alpha, p(.)} \\
& \geq \frac{B_{1}}{\alpha\left(p^{+}\right)^{\alpha}}\left(\sum_{k \in \mathbb{Z}}|\Delta u(k)|^{p(k)}\right)^{\alpha}+\frac{1}{p^{+}} \sum_{k \in \mathbb{Z}}|u(k)|^{p(k)}-C_{7}^{\prime}\|u\|_{1, \alpha, p(.)} \\
& \geq \min \left(\frac{B_{1}}{\alpha\left(p^{+}\right)^{\alpha}} ; \frac{1}{p^{+}}\right)\left(\left(\sum_{k \in \mathbb{Z}}|\Delta u(k)|^{p(k)}\right)^{\alpha}+\sum_{k \in \mathbb{Z}}|u(k)|^{p(k)}\right)-C_{7}^{\prime}\|u\|_{1, \alpha, p(.)} \\
& \geq \min \left(\frac{B_{1}}{\alpha\left(p^{+}\right)^{\alpha}} ; \frac{1}{p^{+}}\right) \rho_{1, \alpha, p(.)}(u)-C_{7}^{\prime}\|u\|_{1, \alpha, p(.)} .
\end{aligned}
$$

To prove the coerciveness of the functional J, we may assume that $\|u\|_{1, \alpha, p(.)}>1$ and, using Proposition 2.8, we deduce from the above inequality that

$$
J(u) \geq \min \left(\frac{B_{1}}{\alpha\left(p^{+}\right)^{\alpha}} ; \frac{1}{p^{+}}\right)\|u\|_{1, \alpha, p(.)}^{p^{-}}-C_{7}^{\prime}\|u\|_{1, \alpha, p(.)} .
$$

Thus,

$$
\mathrm{J}(\mathrm{u}) \longrightarrow+\infty \text { as }\|\mathfrak{u}\|_{1, \alpha, p(.)} \longrightarrow+\infty,
$$


namely $\mathrm{J}$ is coercive.

Besides, for $\|u\|_{1, \alpha, p(.)} \leq 1$, we have

$$
\begin{aligned}
J(u) & \geq \min \left(\frac{B_{1}}{\alpha\left(p^{+}\right)^{\alpha}} ; \frac{1}{p^{+}}\right) \rho_{1, \alpha, p(.)}(u)-C_{7}^{\prime}\|u\|_{1, \alpha, p(.)} \\
& \geq-C_{7}^{\prime}\|u\|_{1, \alpha, p(.)} \\
& >-\infty
\end{aligned}
$$

Thus $\mathrm{J}$ is bounded below.

Since $\mathrm{J}$ is proper, weakly lower semi-continuous and coercive on $\mathcal{W}_{\alpha}^{1, p(.)}$, using the relation between critical points of J and problem (1.1), we deduce that J has a minimizer which is a weak homoclinic solution of (1.1).

\section{An extension}

In this section we consider the following problem

$$
\left(P_{u}\right)\left\{\begin{array}{l}
-M(A(k-1, \Delta u(k-1))) \Delta(a(k-1, \Delta u(k-1))) \\
+|u(k)|^{p(k)-2} u(k)=f(k, u(k)), k \in \mathbb{Z} \\
\lim _{|k| \rightarrow \infty} u(k)=0,
\end{array}\right.
$$

where we assume that

$$
\left\{\begin{array}{l}
f(k, .): \mathbb{R} \longrightarrow \mathbb{R} \text { is a continuous function for all } k \text { in } \mathbb{Z} \\
c: \mathbb{Z} \longrightarrow[0 ;+\infty) \text { is such that } c^{+}<+\infty \text { and }|f(k, t)| \leq c(k)|t|^{\beta(k)-1} \\
\text { with } \beta: \mathbb{Z} \longrightarrow \mathbb{R} \text { such that } 1<p^{-} \leq p^{+}<\beta^{-} \leq \beta^{+}
\end{array}\right.
$$

For any $u \in \mathcal{W}_{\alpha}^{1, p(.)}$, the energy functional corresponding to (4.1) is

$$
J(u)=\widehat{M}\left(\sum_{k \in \mathbb{Z}} A(k-1, \Delta u(k-1))\right)+\sum_{k \in \mathbb{Z}} \frac{1}{p(k)}|u(k)|^{p(k)}-\sum_{k \in \mathbb{Z}} F(k, u(k)),
$$

where $F(k, u(k))=\int_{0}^{u(k)} f(k, t) d t$ 
Definition 4.1. A weak homoclinic solution of problem (4.1) is a function $u \in \mathcal{W}_{\alpha}^{1, \mathfrak{p}(.)}$ such that

$$
\left\{\begin{array}{l}
M\left(\sum_{k \in \mathbb{Z}} A(k-1, \Delta u(k-1))\right) \sum_{k \in \mathbb{Z}} a(k-1, \Delta u(k-1)) \Delta v(k-1) \\
+\sum_{k \in \mathbb{Z}}|u(k)|^{p(k)-2} u(k) v(k)=\sum_{k \in \mathbb{Z}} f(k, u(k)) v(k),
\end{array}\right.
$$

for any $v \in \mathcal{W}_{\alpha}^{1, p(.)}$.

We have the following result:

Theorem 4.2. Under assumptions (2.1)- (2.7) and (4.2), the problem (4.1) has at least one weak solution.

Proof. Let $h(u)=\sum_{k \in \mathbb{Z}} F(k, u(k))$, therefore $h^{\prime}: \mathcal{W}_{\alpha}^{1, p(.)} \longrightarrow \mathcal{W}_{\alpha}^{1, p(.)}$ is completly continuous and thus, $h$ is weakly lower semi-continuous. Then $J \in \mathrm{C}^{1}\left(\mathcal{W}_{\alpha}^{1, p(\cdot)} ; \mathbb{R}\right)$ and is weakly lower semicontinuous.

On the other hand, for all $u, v \in \mathcal{W}_{\alpha}^{1, p(.)}$, we have

$$
\lim _{\delta \rightarrow 0^{+}} \frac{h(u+\delta v)-h(u)}{\delta}=\lim _{\delta \rightarrow 0^{+}} \sum_{k \in \mathbb{Z}} \frac{F(k, u(k)+\delta v(k))-F(k, u(k)}{\delta},
$$

and since

$$
\begin{aligned}
\sum_{k \in \mathbb{Z}}\left|\frac{F(k, u(k)+\delta v(k))-F(k, u(k))}{\delta}\right| \leq & \frac{c^{+}}{\delta} \sum_{k \in \mathbb{Z}}\left(\int_{0}^{\mathfrak{u}(k)+\delta v(k)}|t|^{\beta(k)-1} d t+\int_{0}^{\mathfrak{u}(k)}|t|^{\beta(k)-1} d t\right) \\
& \leq \frac{c^{+}}{\delta \beta^{-}} \sum_{k \in \mathbb{Z}}\left(|u(k)+\delta v(k)|^{\beta(k)}+|u(k)|^{\beta(k)}\right) \\
& <+\infty
\end{aligned}
$$

we obtain

$$
\begin{aligned}
\lim _{\delta \rightarrow 0^{+}} \frac{h(u+\delta v)-h(u)}{\delta} & =\sum_{k \in \mathbb{Z}} \lim _{\delta \rightarrow 0^{+}} \frac{F(k, u(k)+\delta v(k))-F(k, u(k)}{\delta} \\
& =\sum_{k \in \mathbb{Z}} f(k, u(k)) v(k) .
\end{aligned}
$$

Consequently,

$$
\left\{\begin{aligned}
\left\langle J^{\prime}(u), v\right\rangle= & M\left(\sum_{k \in \mathbb{Z}} A(k-1, \Delta u(k-1))\right) \sum_{k \in \mathbb{Z}} a(k-1, \Delta u(k-1)) \Delta v(k-1) \\
& +\sum_{k \in \mathbb{Z}}|\mathfrak{u}(k)|^{p(k)-2} \mathfrak{u}(k) v(k)-\sum_{k \in \mathbb{Z}} f(k, \mathfrak{u}(k)) v(k),
\end{aligned}\right.
$$


for all $u, v \in \mathcal{W}_{\alpha}^{1, p(.)}$.

A critical point to J, i.e. a point $u \in \mathcal{W}_{\alpha}^{1, p(.)}$ such that

$$
\left\langle J^{\prime}(u), v\right\rangle=0 \text { for all } v \in \mathcal{W}_{\alpha}^{1, p(.)}
$$

is a weak solution to (4.1). To end the proof of Theorem 4.2, we have to prove that $\mathrm{J}$ is coercive and bounded from below.

For $u \in \mathcal{W}_{\alpha}^{1, p(.)}$ such that $\|u\|_{1, \alpha, \mathfrak{p}(.)}>1$, we have

$$
\begin{aligned}
J(u) & \geq \min \left(\frac{B_{1}}{\alpha\left(p^{+}\right)^{\alpha}} ; \frac{1}{p^{+}}\right)\|u\|_{1, \alpha, p(.)}^{p^{-}}-\sum_{k \in \mathbb{Z}} F(k, u(k)) \\
& \geq \min \left(\frac{B_{1}}{\alpha\left(p^{+}\right)^{\alpha}} ; \frac{1}{p^{+}}\right)\|u\|_{1, \alpha, p(.)}^{p^{-}}-\sum_{k \in \mathbb{Z}} \int_{0}^{u(k)}|f(k, t)| d t \\
& \geq \min \left(\frac{B_{1}}{\alpha\left(p^{+}\right)^{\alpha}} ; \frac{1}{p^{+}}\right)\|u\|_{1, \alpha, p(.)}^{p^{-}}-\frac{c^{+}}{\beta^{-}} \sum_{k \in \mathbb{Z}}|u(k)|^{\beta(k)} \\
& \geq \min \left(\frac{B_{1}}{\alpha\left(p^{+}\right)^{\alpha}} ; \frac{1}{p^{+}}\right)\|u\|_{1, \alpha, p(.)}^{p^{-}}-\frac{c^{+} k}{\beta^{-}}
\end{aligned}
$$

where $\mathrm{K}$ is a positive constant. As $p^{-}>1$, then $J$ is coercive.

On the other hand, for $u \in \mathcal{W}_{\alpha}^{1, p(.)}$ such that $\|u\|_{1, \alpha, \mathfrak{p}(.)}<1$, we have

$$
\begin{aligned}
J(u) & \geq \min \left(\frac{B_{1}}{\alpha\left(p^{+}\right)^{\alpha}} ; \frac{1}{p^{+}}\right)\|u\|_{1, \alpha, p(.)}^{\alpha p^{+}}-\frac{c^{+} K}{\beta^{-}} \\
& \geq-\frac{c^{+} K}{\beta^{-}} \\
& >-\infty
\end{aligned}
$$

Therefore, $\mathrm{J}$ is bounded from below.

\section{References}

[1] H. Brezis; Analyse Fonctionnelle: Theorie et Applications. Paris, Masson, 1983.

[2] A. Cabada, C. Li, S. Tersian; On homoclinic solutions of a semilinear p-Laplacian difference equation with periodic coefficients. Adv. Difference Equ. 2010, Art. ID 195376, 17 pp.

[3] A. Guiro, B. Kon and S. Ouaro; Weak homoclinic solutions of anisotropic difference equation with variable exponents. Adv. Difference Equ. 154 (2013), 13 pp.

[4] A. Guiro, B. Kon and S. Ouaro; Weak heteroclinic solutions of anisotropic difference equation with variable exponents. Electron. J. Diff. Equ. 225 (2013), 1-9. 
[5] B. Kon and S. Ouaro; Weak solutions for anisotropic discrete boundary value problems. J. Difference Equ. Appl. 17, N.10, (2011), 1537-1547.

[6] B. Kon, S. Ouaro and S. Traor; Weak solutions for anisotropic nonlinear elliptic equations with variable exponent. Electron. J. Diff. Equ. 144 (2009), 1-11.

[7] M. Mihailescu, P. Pucci and V. Radulescu; Nonhomogeneous boundary value problems in anisotropic Sobolev spaces. C. R. Acad. Sci. Paris, Ser. I 345 (2007), 561-566.

[8] M. Mihailescu, P. Pucci and V. Radulescu; Eigenvalue problems for anisotropic quasilinear elliptic equations with variable exponent. J. Math. Anal. Appl. 340 (2008), 687-698.

[9] M. Mihailescu, V. Radulescu and S. Tersian; Eigenvalue problems for anisotropic discrete boundary value problems. J. Difference Equ. Appl. 15(6) (2009), 557-567.

[10] M. Mihailescu, V. Radulescu and S. Tersian; Homoclinic solutions of difference equations with variable exponents. Topol. Methods Nonlinear Anal. 38 (2011) 2, 277-289. 